\title{
Errors in proofreading: Evidence for syntactic control of letter processing?
}

\author{
SHIMON ABRAMOVICI \\ Department of Education, University of Edinburgh, Edinburgh, Scotland
}

\begin{abstract}
The present study compared the rates of detection of misspellings in content and in function words, controlling for the effects of familiarity by studying verbs that could be either content words or function words according to the context. In agreement with previous studies, it was found that errors were more readily detected in content words than in function words, thereby clearly demonstrating the importance of syntactic factors in processing the words in a text. Unlike previous studies, we did not find consistent evidence that errors changing the overall word shape were detected more easily than errors preserving word shape, and we found no evidence that the position of the error in the word affected its rate of detection. These findings were interpreted in the framework of an interactive approach to the reading process.
\end{abstract}

It seems reasonable to assume that performance on proofreading tasks provides a measure of visual attention to letters and words in the text. Therefore, it is not surprising that the analysis of proofreading errors has been one of the methods used to investigate the psychological processes in reading.

A recent sudy of this type (Haber \& Schindler, 1981) reported that subjects reading prose were less likely to detect errors in function words than in content words of the same length. In this study, it was also found that misspellings that changed the shapes of the words in terms of the occurrence of ascending, descending, and short letters were more likely to be detected than those that did not do so; moreover, this effect was larger for function words than for content words. Haber and Schindler thought that this suggested that function words, and perhaps short content words, were often recognized by their overall shape rather than by analysis of individual letters. They went on to discuss three alternative explanations for these differences in strategy of processing. According to the first explanation, function words have a high frequency of occurrence, which leads to great familiarity with them in mature readers, and as a result, these words can be recognized without much attention to detail. The second explanation stresses the fact that the category of function words contains most of the short words in English and mature readers can perhaps recognize such words without much attention to detail. The third account assumes that more predictable words draw less visual attention to their visual letter features than do less predictable words; since the function words of a sentence are more predictable than its content words, the former type of word draws less visual attention from the reader than the latter type of word.

Although these explanations are not mutually exclusive, it would be interesting to separate them in order to determine their relative importance. Haber and Schindler
(1981) proposed that the contribution of familiarity and predictability could be separated by comparing rates of error detection for content words and function words occurring in contexts in which their likelihood of occurrence varied (see Haber \& Schindler, 1981, p. 577). One of the problems with their approach is that the artificiality of their contrived materials is likely to make it impossible to draw valid conclusions about reading from the study. It was the chief aim of the present study to explore an alternative approach to the problem.

Consider the verb "have" in the following pair of sentences: (1) I have a book. (2) I have found a book. In Sentence 1, "have" is the main verb and it functions as a content word meaning possess or own, whereas in Sentence 2, "have" is an auxiliary verb and as such it is a function word (see Clark \& Clark, 1977; Quirk \& Greenbaum, 1973). This example demonstrates that there are verbs that can function either as auxiliary or as full lexical verbs, the former being instances of function words and the latter, of content words. Since the graphic representations of the auxiliary and the lexical verb in such cases are identical, differences in error detection in such pairs of verbs are likely to be a result of syntactic factors rather than of familiarity or length. In the present study, rates of error detection in three such pairs of verbs (i.e., "was," "had," and "have") were investigated. These verbs were studied because a children's story was found containing instances of these verbs used as lexical verbs and as auxiliary verbs. This enabled us to investigate the question in a relatively naturalistic context.

Although the subjects in Haber and Schindler's (1981) study were adults, Haber and Haber (1981) argued that it was important to investigate the role of word shape in reading acquisition. Therefore, in the present study, a group of children was included as well as a group of adults. Some information was collected about the children's reading ability to find out to what 
extent the effects were related to reading proficiency. Apart from the changes discussed above, the present study attempted to replicate Haber and Schindler's (1981) study. There were three main hypotheses or questions. First, if rate of error detection in content and function words were largely determined by frequency and familiarity, we would expect little difference between the auxiliary verbs and the lexical verbs in our study, since the words selected from these categories were graphologically identical. On the other hand, if the effects were largely determined by syntactic factors, we would expect differences in rates of error detection in instances of the two types of verb. Second, if shape were an important factor in the identification of function words, we would expect errors that changed the shapes of words to be detected more easily than errors that preserved the shape, and we would expect the difference in detection rate of the two types of error to be greater in the case of the auxiliary verbs than in the case of lexical verbs. Third, Haber and Schindler (1981) found that the rate of error detection was affected by the position of the error in the word; errors that occurred in the middle of the word were harder to detect than errors occurring at the beginning or the end. This factor was included in the present study as well, and the question was to what extent these findings would replicate. The final set of questions is concerned with the effects of age and reading ability on error detection.

\section{METHOD}

\section{Subjects}

There were two groups of subjects: a group of children and a group of adults. In the first group, there were 199 children; 106 were boys and 93 were girls. Their mean age was 11 years 8 months, and the range of their ages was 10 years 10 months to 12 years 3 months. The second group consisted of 93 adults, 24 males and 69 females. Their mean age was 20 years 4 months, and the range of their ages was 17 years 11 months to 42 years.

\section{Materials}

The text used in this study was the children's story "The Princess Whom Nobody Could Silence" (see Wiggin \& Smith, 1927 ), a story of about 1,000 words.

Twenty-four versions of the story were prepared, each containing 37 errors. They were divided into two sets, with 12 versions in each. In one set of 12 versions, errors were introduced in the auxiliary verbs "was," "had," and "have," whereas in the other set of 12 versions errors were introduced in the lexical verbs "was," "had," and "have." The former set will be referred to as the "function" set and the latter, as the "content" set. To prevent subjects from easily discovering the "target" words, errors were introduced also in 18 words that were not related to the hypothesis of the study. These words and the errors introduced in them were the same in all versions, and they will be referred to as "fillers."

The errors introduced in the target words in six versions of each set preserved the shape of the words, whereas the errors introduccd in the remaining six changed word shape in terms of the occurtence of ascending, descending, and short letters, as in Haber and Schindler's (1981) study. None of the errors introduced formed other correctly spelled words. The six ways of ordering the three positions (beginning, center, and end) were used to assign the three target words to different positions of errors and thereby to generate the different versions. For example, in one version, errors were introduced in the first letter in instances of "was," in the second letter in instances of "had," and in the last letter, in instances of "have."

The same method was used to generate both the "function" versions and the "content" versions, so that for each version in one set there was a parallel version in the other set. In the case of the word "have," identical errors were introduced in these parallel versions; this was possible because there were 10 instances of the auxiliary verb "have" and 10 instances of the lexical verb "have" in the study. In the case of the verbs "was" and "had," there were more instances of the auxiliary verb than of the lexical verb (three vs. two and six vs. two, respectively). To keep the number of errors the same in all versions, five errors were introduced in five other three-letter words in the content set, and the results pertaining to them were not included in the analyses.

The assignment of specific errors to versions in the function set was randomized. Repetition of the same error within a version was avoided as far as possible.

In addition to these versions of the text, a 10-question multiple-choice questionnaire was devised. Seven of the questions in this questionnaire tested comprehension of the details and sequence of events in the story, and the remaining three questions tested the subjects' knowledge of the spellings of the words "was," "had," and "have." In each case, the correct word was given in a sentence frame together with a number of misspellings, the subjects' task being to underline the correct form of the word.

A list of the words in which errors occurred was prepared and distributed among the children at the end of the experiment to enable them to verify the spellings of words about which they were unsure.

\section{Design}

The results for each word were treated separately. The following factors were included in the analyses: age group, type of word (lexical verb, auxiliary verb), position of error (beginning, center, end) and type of crror (preserving shape, altering shapc). In the case of the children, information was collected from the teachers on each child's reading ability; cach child was assigned to one of the following categories: very good reader, good reader, poot reader, very poor (remedial) reader. This information was included in the appropriate analyses. However, since age and reading ability did not have any important interactions with the remaining factors, they will not be further discussed.

\section{Procedure}

Subjects were run in groups. Booklets containing the different versions of the text were randomly mixed and then distributed to the subjects. The experimenter read the instructions while the subjects followed them in their booklets. The instructions stressed that the subjects were to read the story only once at their normal pace, trying to understand it, as a comprehension test was to follow, and that they were to underline errors that they noticed while they were reading.

At the end of the passage, an instruction was printed requesting the subject to put up his/her hand. When a subject indicated that he/she had finished reading the text, the booklet with the story was removed and the comprehension test was given.

When the children completed the comprehension test, a list of the words in which errors occurred was given and they were encouraged to verify the spellings of words about which they were unsure.

\section{RESULTS}

The two main dependent measures were the rate of error detection in each of the words "was," "had," and "have" and performance on the comprehension test. 


\section{Error Detection}

The number of errors detected by each subject in each word was converted into a percentage of the total number of misspellings for that word. For each of the words "was" and "had," these percentages were calculated once including all the instances of error and once excluding the extra errors in the function set. Various analyses of variance were performed on these scores in which the factors were type of word, position, and type of error. In one set of analyses, the sample of errors of each type for each position in each target word was divided into two independent subsets and a factor of the set of errors was included in the analyses with the aim of investigating whether the findings were dependent on the sample of misspellings used.

The results of all the analyses were in very close agreement. The main findings are summarized in Table $1 .^{1}$

In Table 1, the word(s) to which each set of analyses refers are given at the top of the table. The relevant factors are listed in the column at the extreme left of the table and the relevant $F$ ratios, with their probabilities, degrees of freedom, and means, are presented for each word.

The effect of the type-of-word factor was significant in all three target words, with a larger proportion of errors on the average being detected in the lexical verbs than in the auxiliary verbs. Evidence that errors that changed the shape of the word were detected better than errors not changing the shape of the word was obtained only with the word "was," and even in this case there was no evidence that this factor interacted with the type-of-word factor. The remaining factors, position and set of errors, did not reach significance with any of the words.

\section{Comprehension}

The results of the comprehension test revealed no significant effects; in fact, the mean of the children's scores and that of the adults were virtually identical (6.00 vs. 6.06). The standard deviations of these means were 1.26 and 1.47 , respectively. These results demonstrate that our subjects read the text for meaning, and they will not be discussed any further.

\section{DISCUSSION}

The main finding of our study was that, in agreement with Haber and Schindler's (1981) study, errors in lexical verbs were more readily detected than errors in auxiliary verbs, and since the pairs of verbs we com. pared were graphologically identical, there can be no doubt that the effect was largely due to syntactic factors. ${ }^{2}$ In addition, since we found the effect consistently with three different pairs of words in which the misspellings were different, it seems fairly safe to generalize these findings beyond the specific words and errors included in the study. Furthermore, the absence of interactions of the set of errors with the type of verb and the observation that the additional errors in the function set did not affect the results both give further support to this claim. It is more difficult to give a definite answer to the question of whether the effect is likely to generalize to other types of content and function words, since only one type was included in our study and Haber and Schindler did not provide information about the types of content and function words in their study, although presumably it included more than one type. This question probably deserves further research.

We did not find consistent evidence for the effects of shape. We found evidence for the effect of this factor on error detection only with the target word "was," and even in this case there was no evidence in the form of an interaction showing that the effect was stronger with the auxiliary verb than with the lexical verb, as one would expect on the basis of Haber and Schindler's (1981) results. Moreover, since this factor was involved in two of the three significant interactions with the set

Table 1

Summary of Main Error Detection Results

\begin{tabular}{|c|c|c|c|c|c|c|c|c|c|c|c|c|c|c|c|c|c|c|}
\hline \multirow[b]{4}{*}{ Factor } & \multicolumn{18}{|c|}{ Word } \\
\hline & \multicolumn{6}{|c|}{ Was } & \multicolumn{6}{|c|}{$\mathrm{Had}$} & \multicolumn{6}{|c|}{ Have } \\
\hline & \multirow[b]{2}{*}{$\mathrm{F}$} & \multirow[b]{2}{*}{$\mathrm{df}$} & \multicolumn{4}{|c|}{ Mean } & \multirow[b]{2}{*}{$\mathrm{F}$} & \multirow[b]{2}{*}{ df } & \multicolumn{4}{|c|}{ Mean } & \multirow[b]{2}{*}{$\mathrm{F}$} & \multirow[b]{2}{*}{ df } & \multicolumn{4}{|c|}{ Mean } \\
\hline & & & $\mathrm{L}$ & A & $\mathbf{S}$ & D & & & $\mathbf{L}$ & A & $\mathbf{S}$ & D & & & $\mathrm{L}$ & A & $S$ & $\mathrm{D}$ \\
\hline Type of Word (TOW) & $30.94^{*}$ & 1,242 & 78 & 56 & & & $17.49^{*}$ & 1,244 & 95 & 81 & & & $9.63 \dagger$ & 1,242 & 89 & 82 & & \\
\hline Position & $1.86 \dagger \dagger$ & 2,242 & & & & & $.42 \dagger \dagger$ & & & & & & $.50 \dagger \dagger$ & & & & & \\
\hline Set of Error & $.08+\dagger$ & 1,242 & & & & & $.09+\dagger$ & & & & & & $.22+\dagger$ & & & & & \\
\hline Type of Error (TOE) & $13.30^{* *}$ & 1,242 & & & 59 & 74 & $1.38 \dagger \dagger$ & 1,244 & & & 86 & 89 & $1.68 \dagger \dagger$ & 1,242 & & & 84 & 87 \\
\hline TOE by TOW & $1.15+\dagger$ & 1,242 & & & & & $.18 \dagger \dagger$ & 1,244 & & & & & $.68 \dagger \dagger$ & 1,242 & & & & \\
\hline $\mathrm{S}$ & & & 68 & 50 & & & & & 92 & 80 & & & & & 88 & 80 & & \\
\hline $\mathrm{D}$ & & & 86 & 62 & & & & & 97 & 81 & & & & & 90 & 84 & & \\
\hline
\end{tabular}

Note-For type of word, $L=$ lexical, $A=$ auxiliary; for type of error, $S=$ similar, $D=$ different. The degrees of freedom for "had" are different from those for "was" and "have" because the results of two children who failed to demonstrate knowledge of the spelling of "was" were removed from the analysis of that word, and for the same reason, the results of two children were removed from the analysis of the word "have." $\quad * p<.0001 . \quad * * p<.0005 . \quad+p<.005$. H+Nonsignificant. 
of errors ${ }^{1}$, it may be the case that evidence for the operation of this factor depends very strongly on the specific misspellings used in the study and that, therefore, in itself, it does not reveal anything about reading. Even if one does not accept the latter argument, the conclusion remains that our results do not support the claim that the overall shape of words plays an important role in reading.

We failed to replicate the positional effects altogether. In demonstrating this effect, Haber and Schindler's (1981) study was in agreement with Sloboda's (1976) study and with Healy's (1980) study. However, Corcoran and Weening (1968), commenting on Corcoran's (1966, 1967) two earlier studies, in which positional effects occurred, observed that the direction of the positional effect that was obtained varied and depended very heavily on the precise nature of the experimental task. Moreover, in four of the six studies just mentioned that obtained positional effects, reading times were measured, and thus perhaps an element of speed was introduced. In the fifth, Sloboda's, study, subjects had both to mark errors and to read the passage aloud into a tape recorder. Taken together, these studies may suggest that positional effects occur more readily when the proofreading task is performed under a certain amount of pressure, whereas in more relaxed reading situations such as that which existed in our study, they are less likely to occur.

To recapitulate, the only effect that replicated consistently was that of the type of word. If we accept the assumption that different rates of error detection reflect depth of processing at the letter level, it follows that we have uncovered some differences in the way lexical and auxiliary verbs are processed. Since we controlled the words for familiarity, it follows that these differences are largely due to syntactic factors. This finding is consistent with an interactive model of reading (see Levy, 1981; Rummelhart, 1977). This type of model claims that the reader employs different processors for different levels of linguistic representation in parallel and the output of each stage in the analysis acts as a data base for all levels. For example, the hypothesis for "was" at the lexical level would be supported by a hypothesis for "w" at the letter level, as well as the expectation for a verb at the syntactic level. It would appear, however, that word shape as we have defined it may not be an important factor in this process.

\section{REFERENCES}

Clark, H. H., \& Clark, E. V. Psychology and language. New York: Harcourt Brace Jovanovich, 1977.

Corcoran, D. W. J. An acoustic factor in letter cancellation. Nature, 1966, 210, 658.

Corcoran, D. W. J. Acoustic factors in proof-reading. Nature, $1967,214,851-852$.

Corcoran, D. W. J., \& Weening, D. L. Acoustic factors in visual search. Quarterly Journal of Experimental Psychology, $1968,20,83-85$.

HABER, N. H., \& HABER, L. R. The shape of a word can specify its meaning. Reading Research Quarterly, 1981, 16, 334-345.

Haber, N. H., \& Schindler, R. M. Errors in proof-reading: Evidence of syntactic control of letter processing. Journal of Experimental Psychology: Human Perception and Performance, 1981, 7, 573-579.

Healy, A. F. Proof-reading errors on the word the: New evidence on reading units. Journal of Experimental Psychology: Human Perception and Performance, 1980, 6, 45-57.

Levy, B. A. Interactive processing during reading. In A. $\mathbf{M}$. Lesgold \& C. A. Perfetti (Eds.), Interactive processes in reading. Hillsdale, N.J: Erlbaum, 1981.

Quirk, R., \& Greenbaum, S. A university grammar of English. London: Longman, 1973.

RUMmelhaRT, D. E. Toward an interactive model of reading. In S. Dornic \& P. M. A. Rabbitt (Eds.), Attention and performance VI. Hillsdale, N. J: Erlbaum, 1977.

Sloboda, J. A. The effect of item position on the likelihood of identification by inference in prose reading and musical reading. Canadian Journal of Psychology, 1976, 30, 228-237.

Wiggin, K. D., \& Smith, N. A. (Eds.). Tales of laughter. New York: Doubleday, 1927.

\section{NOTES}

1. With the exception of the interaction of type of word and type of error, only main effects were included in Table 1 . In addition to these, the set-of-errors factor had a number of significant three-term interactions. These were not discussed in detail because the remaining factors in these interactions varied across the words. They are listed here. For the word "was," there was an interaction of Set of Error by Type of Error by Position, for the word "had," there was an interaction of Set of Error by Age by Type of Word, and for the word "have," there was an interaction of Set of Error by Type of Error by Age.

2. We have, in fact, demonstrated empirically that the auxiliary verbs "was," "had," and "have" in our study were more predictable than the same lexical verbs. In a cloze passage administered to 701 st-year students, the mean percentages for the occurrence of the target word for the auxiliary verbs "was," "had," and "have" were $91.25 \%, 97.57 \%$, and $99.14 \%$, respectively. The mean percentages for the same lexical verbs were $77.14 \%, 44.28 \%$, and $80.00 \%$. In each case, the difference between the means was significant.

(Received for publication August 26, 1982; revision accepted February 1, 1983.) 